disease or nonfatal myocardial infarction. Secondary endpoints included stroke, death from any cause, and combined cardiovascular disease.

The effect on primary outcome of both lisinopril and amlodipine was comparable to that of chlortalidone, with no significant difference between blacks and nonblacks. For lisinopril vs chlortalidone, racial differences were seen with regard to the following secondary outcomes: blood pressure (larger reduction in blacks given chlortalidone vs nonblacks), stroke, and combined cardiovascular disease outcomes. Black patients given chlortalidone had a lower relative risk of stroke than those receiving lisinopril. The only significant difference between amlodipine and chlortalidone was seen in heart failure (lower relative risk in chlortalidone-treated patients), but this was consistent between racial subgroups.

The authors conclude that, because of the lack of superiority of amlodipine or lisinopril to chlortalidone in both black and nonblack patients, thiazide diuretics should remain the treatment of choice for all hypertensive patients.

Original article Wright JT Jr et al. (2005) Outcomes in hypertensive black and nonblack patients treated with chlorthalidone, amlodipine, and lisinopril. JAMA 293: 1595-1608

\section{Novel anticoagulant therapy: safety and pharmacokinetics}

Results from the first human trial of Sunol-cH36 have recently been reported in the European Heart Journal. This chimeric monoclonal antibody to tissue factor inhibits the formation of thrombin by blocking the production of factors $\mathrm{Xa}$ and IXa, and is being investigated as a novel anticoagulant therapy.

The PROXIMATE-TIMI 27 trial, carried out at four US centers, included 26 patients with stable coronary artery disease. In an open-label, dose-escalation design, patients received a single intravenous bolus of Sunol-cH36 at a dose of $0.03,0.06,0.08,0.10$ or $0.30 \mathrm{mg} / \mathrm{kg}$, and once-daily aspirin (81-325 mg). Patients were observed for $8 \mathrm{~h}$ after administration of Sunol-cH36. They then attended follow-up visits for the next 3 days and then at intervals for 7 weeks.
No major bleeding events were observed during the study, although spontaneous minor bleeding occurred in 14 patients, usually within 3 days of receiving the bolus. These events appeared to be related to the dose of Sunol-cH36, and were mostly consistent with platelet-mediated bleeding (e.g. spontaneous tongue hematomas or oral mucosal bleeding). No serious adverse events were reported and the median terminal half-life of the drug was $72.2 \mathrm{~h}$.

Observing that the dose-dependent anticoagulant effects of Sunol-cH36 might be clinically important, Morrow and co-investigators suggest that further studies are warranted.

Original article Morrow DA et al. (2005) Potent inhibition of thrombin with a monoclonal antibody against tissue factor (Sunol-cH36): results of the PROXIMATE-TIMI 27 trial. Eur Heart J26: 682-688

\section{Renalase regulates blood pressure and cardiac function}

A recent study by $X u$ et al. has revealed that renalase, a novel flavin adenine dinucleotidedependent amine oxidase secreted by the kidney, appears to regulate cardiac function and systemic blood pressure.

The authors hypothesized that the kidney might secrete biologically important proteins other than those described thus far, and they searched for such proteins by analyzing all the clones published by the Mammalian Gene Collection Project. Of 114 candidate genes identified, one gene exhibited robust and preferential expression in human kidney, although its expression was also detected in the heart, skeletal muscle and small intestine. Naming the gene 'renalase', the team went on to demonstrate that its product is readily detectable in human plasma; furthermore, patients with end-stage renal disease showed lower plasma concentrations of renalase than healthy individuals.

Having determined that renalase specifically metabolizes catecholamines (dopamine, epinephrine and norepinephrine), Xu and colleagues studied its effects on cardiovascular hemodynamics. In a rat study, a single bolus injection of the protein was associated with rapid, transient, and dose-dependent decrease in systolic, diastolic and mean 